\title{
Coffee, CYP1A2 genotype and risk of myocardial infarction
}

\author{
Ahmed El-Sohemy • Marilyn C. Cornelis • \\ Edmond K. Kabagambe $\cdot$ Hannia Campos
}

Published online: 21 September 2007

(C) Springer-Verlag and NuGO 2007

Keywords Coffee - Caffeine - CYP1A2 .

Myocardial infarction $\cdot$ Nutrigenetics

The association between coffee intake and risk of myocardial infarction (MI) remains controversial [1, 2]. Coffee is a major source of caffeine, which is metabolized by the polymorphic CYP1A2 enzyme. An A to C substitution at position $734(C Y P 1 A 2 * 1 F)$ in the CYPlA2 gene decreases enzyme inducibility as measured by plasma or urinary [caffeine]/[caffeine metabolite] ratio after a dose of caffeine, resulting in impaired caffeine metabolism [3]. Individuals who are homozygous for the $C Y P 1 A 2 * 1 A$ allele (A/A) are "rapid" caffeine metabolizers whereas carriers of the variant $C Y P 1 A 2 * 1 F$ are "slow" caffeine metabolizers. The objective of this study was to determine whether CYP1A2 genotype modifies the association between coffee consumption and risk of MI. Cases $(n=2,014)$ with a first acute non-fatal MI and population-based controls $(n=2,014)$ were genotyped by RFLP-PCR. A food

A. El-Sohemy $(\square) \cdot$ M. C. Cornelis Department of Nutritional Sciences, University of Toronto,

Toronto, Canada

e-mail: a.el.sohemy@utoronto.ca

E. K. Kabagambe

Department of Nutrition,

Harvard School of Public Health,

Boston, USA

H. Campos

Centro Centroamericano de Poblacion,

Universidad de Costa Rica,

San Pedro de Montes de Oca, Costa Rica frequency questionnaire was used to assess coffee intake. Odds ratios (OR) and 95\% confidence intervals (CI) were calculated using conditional logistic regression. For carriers of the slow $* 1 F$ allele, the ORs $(95 \% \mathrm{CI})$ for risk of MI associated with consuming $<1,1,2-3$ and 4 or more cups/ day were 1.00 (reference), 0.99 (0.69-1.44), 1.36 (1.011.83), and 1.64 (1.14-2.34), respectively. Corresponding ORs $(95 \% \mathrm{CI})$ for individuals with the rapid $* 1 A / * 1 A$ genotype were $1.00,0.75(0.51-1.12), 0.78(0.56-1.09)$, and $0.99(0.66-1.48)(P=0.04$ for gene-diet interaction). It has previously been suggested that coffee may be associated with an increased risk of MI only among younger

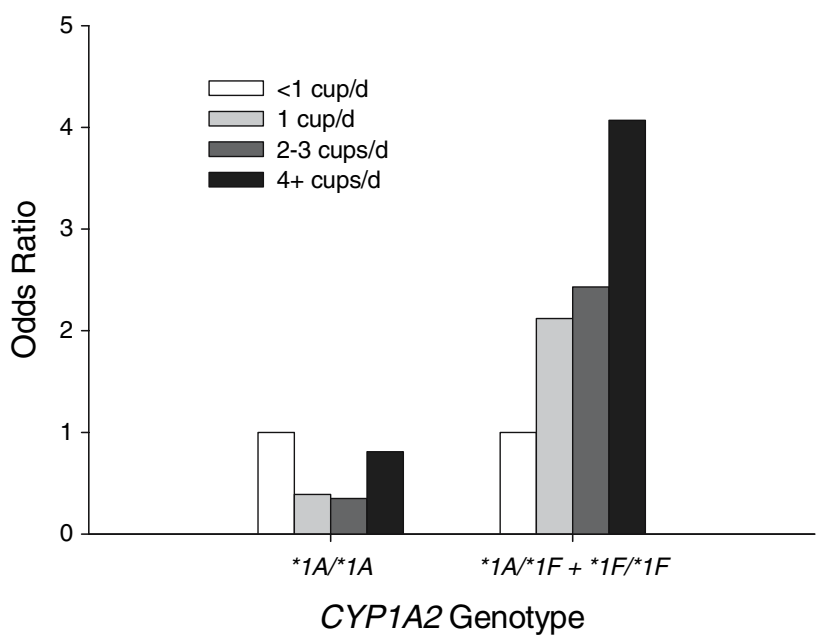

Fig. 1 Coffee intake and risk of MI by CYP1A2 genotype among subjects less than 50 years of age. Adjusted for age, sex, area of residence, smoking (never, past, 1-19 cigarettes/day, $\geq 20$ cigarettes/ day), waist-to-hip ratio, income, physical activity, history of diabetes, history of hypertension and intake of total energy, saturated fat intake, polyunsaturated fat, trans fat, sucrose and alcohol 
individuals. Thus, we examined the effects of coffee among subjects below the age of 50 years. For carriers of the $* I F$ allele the ORs $(95 \% \mathrm{CI})$ of MI associated with consuming $<1,1,2-3$, or $\geq 4$ cups/day of coffee were 1.00 , 2.12 (0.86-5.24), 2.43 (1.22-4.82), and 4.07 (1.89-8.74), respectively (Fig. 1). Corresponding ORs (95\% CI) for those with the $* 1 A / * 1 A$ genotype were $1.00,0.39(0.15-$ $0.97), 0.35$ (0.17-0.76), and $0.81(0.32-2.05)(P<0.001$ for gene-coffee interaction). The protective effects observed among rapid metabolizers suggest that the efficient elimination of caffeine might have unmasked the protective effects of other chemicals in coffee. Compounds in coffee such as caffeic acid and chlorogenic acid have antioxidant properties that might protect against heart disease [4]. In summary, intake of coffee was associated with an increased risk of MI only among those with impaired caffeine metabolism, suggesting that caffeine plays a major role in this association.
Acknowledgments This research was supported by grants from the Canadian Institutes of Health Research (grant No. MOP-53147), and the National Institutes of Health (grant No. HL 60692 and HL 071888). Ahmed El-Sohemy holds a Canada Research Chair in Nutrigenomics.

\section{References}

1. Greenland S (1993) A meta-analysis of coffee, myocardial infarction, and coronary death. Epidemiology 4(4):366-374

2. Kawachi I, Colditz GA, Stone CB (1994) Does coffee drinking increase the risk of coronary heart disease? Results from a metaanalysis. Br Heart J 72(3):269-275

3. Sachse C, Brockmoller J, Bauer S, Roots I (1999) Functional significance of a $\mathrm{C}->\mathrm{A}$ polymorphism in intron 1 of the cytochrome P450 CYP1A2 gene tested with caffeine. Br J Clin Pharmacol 47(4):445-449

4. Daglia M, Papetti A, Gregotti C, Berte F, Gazzani G (2000) In vitro antioxidant and ex vivo protective activities of green and roasted coffee. J Agric Food Chem 48(5):1449-1454 